\title{
Heart Rate Changes Following the Administration of Sugammadex to Infants and Children With Comorbid Cardiac, Cardiovascular, and Congenital Heart Diseases
}

\author{
Jordan Arends a, c, f, Richard Hubbard ${ }^{\mathrm{b}}$, Shabana Z. Shafyc, Mohammed Hakim ${ }^{\mathrm{c}}$, \\ Stephani S. Kimc, Dmitry Tumin ${ }^{\text {d }}$, Joseph D. Tobias ${ }^{c, ~ e ~}$
}

\begin{abstract}
Background: Sugammadex is a novel, rapidly-acting pharmacologic agent to reverse steroidal neuromuscular blocking agents with demonstrated advantages over acetylcholinesterase inhibitors. However, anecdotal reports have noted rare instances of bradycardia and even cardiac arrest. The current study examined heart rate (HR) changes in infants and children with comorbid cardiac, cardiovascular, and congenital heart diseases.
\end{abstract}

Methods: Patients less than 18 years of age, who had a comorbid cardiac, cardiovascular, or congenital heart disease and were to receive sugammadex, were included in this prospective observational study. After sugammadex administration, HR was continuously monitored and recorded every minute for the first $15 \mathrm{~min}$, and then every 5 min for the next 15 min or until the patient was transferred from the operating room. The primary outcome, bradycardia, was defined as HR below the fifth percentile for age. Secondary outcomes included greatest decrease in HR from baseline for each patient and interventions required for bradycardia.

Results: The study cohort included 99 patients (58 male and 41 female) with a median age of 3 years. Bradycardia was noted in 20 of 99 patients (20\%); however, six of these patients were bradycardic prior to the administration of sugammadex. Older patients, male pa-

Manuscript submitted February 26, 2020, accepted June 23, 2020

Published online August 1, 2020

aOhio University Heritage College of Osteopathic Medicine, Dublin, OH, USA

${ }^{b}$ Department of Anesthesiology, University of Texas, Houston, TX, USA

'Department of Anesthesiology and Pain Medicine, Nationwide Children's Hospital, Columbus, OH, USA

${ }^{\mathrm{d}}$ Department of Pediatrics, Brody School of Medicine at East Carolina University, Greenville, NC, USA

eDepartment of Anesthesiology and Pain Medicine, The Ohio State University College of Medicine, Columbus, OH, USA

${ }^{f}$ Corresponding Author: Jordan Arends, Department of Anesthesiology and Pain Medicine, Nationwide Children's Hospital, 700 Children's Drive, Columbus, OH 43205, USA. Email: ja555516@ohio.edu

doi: https://doi.org/10.14740/cr1045 tients, and patients with higher body weight were the most likely to experience bradycardia. None of the patients required treatment for bradycardia.

Conclusions: The incidence of bradycardia following the administration of sugammadex was low, even in patients with congenital heart disease. Bradycardia was not associated with clinically significant hemodynamic changes and no treatment was required.

Keywords: Sugammadex; Heart rate; Bradycardia

\section{Introduction}

Sugammadex is a novel fast-acting agent used to reverse the steroidal neuromuscular blocking agents (NMBAs), rocuronium and vecuronium [1-5]. The US Food and Drug Administration (FDA) approved its use in adults in December 2015 as the first non-competitive antagonist for the reversal of neuromuscular blockade (NMB). In pre-clinical trials, the majority of reported adverse effects were minor and self-limited; however, there were reports of marked bradycardia, with occasional progression to cardiac arrest within minutes after its administration [6]. Despite concerns regarding the possibility of bradycardia and anecdotal reports of its occurrence, the causal relationship between sugammadex and bradycardia remains speculative as no definitive mechanism has been proposed [7-9].

We previously conducted a prospective observational study in a cohort of 221 patients to define the incidence of bradycardia in a general pediatric population requiring anesthetic care [10]. Bradycardia, defined as heart rate (HR) less than the fifth percentile for age, was noted in 18 patients $(8 \%$; $95 \%$ confidence interval (CI): $5 \%, 13 \%$ ), occurring at a median of 2 min after the administration of sugammadex. Among patients developing bradycardia, seven of 18 (38\%) had comorbid cardiac conditions including congenital heart disease. Despite the $8 \%$ incidence of bradycardia, no associated hemodynamic concerns including hypotension were noted, and none of the 18 patients required treatment with vasoactive medications, anticholinergic agents, or fluid. The current study 


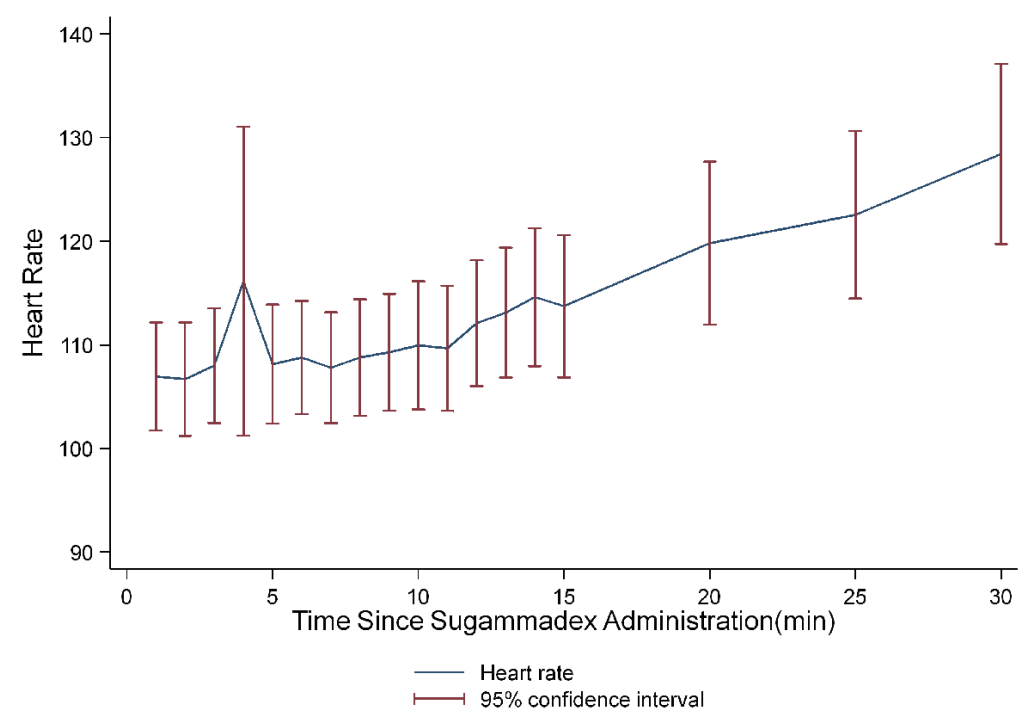

Figure 1. Average heart rate for the first $30 \mathrm{~min}$ following sugammadex administration $(n=92)$. Seven patients with bradycardia at baseline were eliminated.

expands on this previous cohort and attempts to define the incidence of bradycardia specifically among infants and children with comorbid cardiac, cardiovascular, and congenital heart diseases. Our primary aim was to describe the incidence of bradycardia in this specific patient population following the administration of sugammadex. Secondarily, we aimed to define the characteristics of patients and procedures associated with bradycardia after sugammadex, and to determine if intervention for the bradycardia was necessary due to clinically significant hemodynamic concerns.

\section{Materials and Methods}

We enrolled infants and children with comorbid cardiac, cardiovascular, and congenital heart diseases who were to receive sugammadex to reverse NMB with rocuronium or vecuronium. The study was approved by the Institutional Review Board of Nationwide Children's Hospital. As an observational study with no randomization, change in clinical care, and the addition of no added risk to the patients, the need to obtain consent was waived. The study was pre-registered at clinicaltrials.gov (NCT03294018). This study was conducted in compliance with the ethical standards of the responsible institution on human subjects. We excluded patients with a known allergy to sugammadex and those greater than 18 years of age. The decision to use sugammadex was based on the clinical judgment of the anesthesia team. After sugammadex administration, we continuously monitored HR and prospectively recorded it every minute for the first $15 \mathrm{~min}$, and then every $5 \mathrm{~min}$ for the next $15 \mathrm{~min}$ or until the patient was transferred from the operating room. We defined bradycardia as HR below the fifth percentile for age [11]. The occurrence of bradycardia, associated hemodynamic compromise including hypotension, the decision to treat bradycardia, and the medications used were prospectively recorded. Additionally, we collected demographic informa- tion, including age, weight, gender, comorbid conditions, type of surgery, and concomitant medications.

Demographic characteristics were reported as a count and percentage for categorical variables and median with interquartile range (IQR) for continuous variables. We compared patient and procedural characteristics by occurrence of bradycardia using Fisher's exact tests for categorical measures, and rank-sum tests for continuous measures. In addition to estimating the incidence of bradycardia, we performed multivariable logistic regression analysis to assess the independent association of patient and procedural characteristics with the onset of bradycardia. We included gender, age, weight, and initial sugammadex dose using used forward selection. Analysis was performed using SAS 9.4 (Cary, NC) and Stata/IC 14.2 (College Station, TX, StataCorp LP), and two-tailed P $<0.05$ was considered statistically significant.

\section{Results}

The study cohort included 99 patients with comorbid cardiac, cardiovascular, and congenital heart diseases, 41 of whom were included in our previous study [10]. The sample included 58 male and 41 female patients with a median age of 3 years (IQR: 0,10 years) and a median weight of $13.5 \mathrm{~kg}$ (IQR: 6.5 , $30.7 \mathrm{~kg}$ ). Initial sugammadex doses ranged from 0.5 to 7.76 $\mathrm{mg} / \mathrm{kg}$ (median $=3.9 \mathrm{mg} / \mathrm{kg}$, IQR: $2.1,4.0 \mathrm{mg} / \mathrm{kg}$ ). A second sugammadex dose was administered to four patients. One patient received a third dose. Procedures included cardiac surgery $(45 \%)$, non-cardiac surgery $(5 \%)$, cardiac catheterization procedures $(29 \%)$, and electrophysiology interventions $(20 \%)$.

Average HR following sugammadex administration is shown in Figure 1. Bradycardia was noted in 20 of 99 patients $(20 \%)$ after the administration of sugammadex; however, six of the patients had bradycardia at baseline resulting in an adjusted incidence of $14 \%$. The HR changes in these 20 patients 
Table 1. Bradycardia in Patients After Sugammadex Administration

\begin{tabular}{|c|c|c|c|c|c|c|}
\hline Patient & $\begin{array}{l}\text { Definition of } \\
\text { bradycardia for the } \\
\text { patient (beats/min) }\end{array}$ & $\begin{array}{l}\text { Baseline heart } \\
\text { rate (beats/min) }\end{array}$ & $\begin{array}{l}\text { Bradycardic } \\
\text { at baseline }\end{array}$ & $\begin{array}{l}\text { Lowest heart rate after } \\
\text { sugammadex (beats/min) }\end{array}$ & $\begin{array}{l}\text { Bradycardic } \\
\text { after } \\
\text { sugammadex }\end{array}$ & $\begin{array}{l}\text { Absolute difference } \\
\text { in heart rate from } \\
\text { baseline (beats/min) }\end{array}$ \\
\hline 1 & $\leq 61$ & 56 & Yes & 57 & Yes & 1 \\
\hline 2 & $\leq 92$ & 91 & Yes & 87 & Yes & 4 \\
\hline 3 & $\leq 82$ & 73 & Yes & 73 & Yes & 0 \\
\hline 4 & $\leq 87$ & 87 & Yes & 87 & Yes & 0 \\
\hline 6 & $\leq 108$ & 91 & Yes & 90 & Yes & 1 \\
\hline 7 & $\leq 66$ & 71 & No & 62 & Yes & 9 \\
\hline 8 & $\leq 66$ & 69 & No & 56 & Yes & 13 \\
\hline 9 & $\leq 61$ & 79 & No & 46 & Yes & 33 \\
\hline 13 & $\leq 77$ & 82 & No & 71 & Yes & 11 \\
\hline 14 & $\leq 57$ & 73 & No & 53 & Yes & 20 \\
\hline 15 & $\leq 108$ & 135 & No & 76 & Yes & 59 \\
\hline 16 & $\leq 61$ & 70 & No & 44 & Yes & 26 \\
\hline 17 & $\leq 61$ & 99 & No & 51 & Yes & 48 \\
\hline 18 & $\leq 108$ & 110 & No & 108 & Yes & 2 \\
\hline 19 & $\leq 66$ & 68 & No & 64 & Yes & 4 \\
\hline 20 & $\leq 82$ & 83 & No & 79 & Yes & 4 \\
\hline
\end{tabular}

are listed in Table 1. There was one patient who was bradycardic at baseline, but was not bradycardic after the administration sugammadex. The seven patients who were bradycardic at baseline were removed from the analysis $(\mathrm{n}=92)$. The decrease in HR was $\geq 20$ beats/min in five patients, with a maximum decrease of 59 beats/min in one patient whose HR decreased from a baseline of 135 to 76 beats $/ \mathrm{min}$. The median baseline HR in the patients who were bradycardic after sugammadex was 82 beats/min (IQR: 71, 109 beats/min) compared to 113 beats/min (IQR: 91,133 beats/min) in the patients who did not develop bradycardia $(\mathrm{P}=0.003)$. The median decrease in HR for those who developed bradycardia was 12 beats/min (IQR: 4, 26 beats/min) compared to four beats/min (IQR: 1, 8 beats $/ \mathrm{min})$ in the non-bradycardic group $(\mathrm{P}=0.006)$. There was no statistically significant difference in sugammadex dose between the two groups $(\mathrm{P}=0.243)$. Patient characteristics are summarized in Table 2 according to the occurrence of bradycardia. No patient required treatment for bradycardia with an anticholinergic agent, vasoactive agent, or fluid administration.

Older patients, male patients, and patients with a greater weight were the more likely to experience bradycardia according to bivariate analysis (Table 2). Bradycardia was also more common in patients who were bradycardic at baseline prior to the administration of sugammadex. Other patient characteristics did not reach a statistically significant association with bradycardia occurrence. Multivariable analysis showed no significant associations between patient or procedural factors and bradycardia risk (Table 3 ). Table 4 summarizes cardiac comorbid conditions for all study patients, and Figure 2 displays average HRs following sugammadex administration by comorbid cardiovascular disease status. Lastly, we observed differences in medications used between those who experienced bradycardia and those who did not. Seven (50\%) of the bradycardic patients received propofol compared to $18(23 \%)$ of the non-bradycardic patients $(\mathrm{P}=0.051)$.

\section{Discussion}

Acetylcholinesterase inhibitors have been used for the reversal of NMB since the 1950s. Despite their efficacy, reported adverse effects include hypersalivation, bronchospasm, bradycardia, and residual blockade. Sugammadex, a synthetically modified cyclodextrin, is a novel pharmacologic agent for reversal of NMB, having a chemical structure with a hydrophilic exterior and a hydrophobic core, which encapsulates vecuronium or rocuronium and thereby reversing NMB [12]. During pre-clinical trials with sugammadex, the majority of reported adverse effects were minor and self-limited with a lower incidence than acetylcholinesterase inhibitors [13-15]. Most importantly, the potential for residual NMB and its clinical sequelae have been shown to be less with sugammadex $[16,17]$. 
Table 2. Patient and Procedural Characteristics According to Occurrence of Bradycardiaa

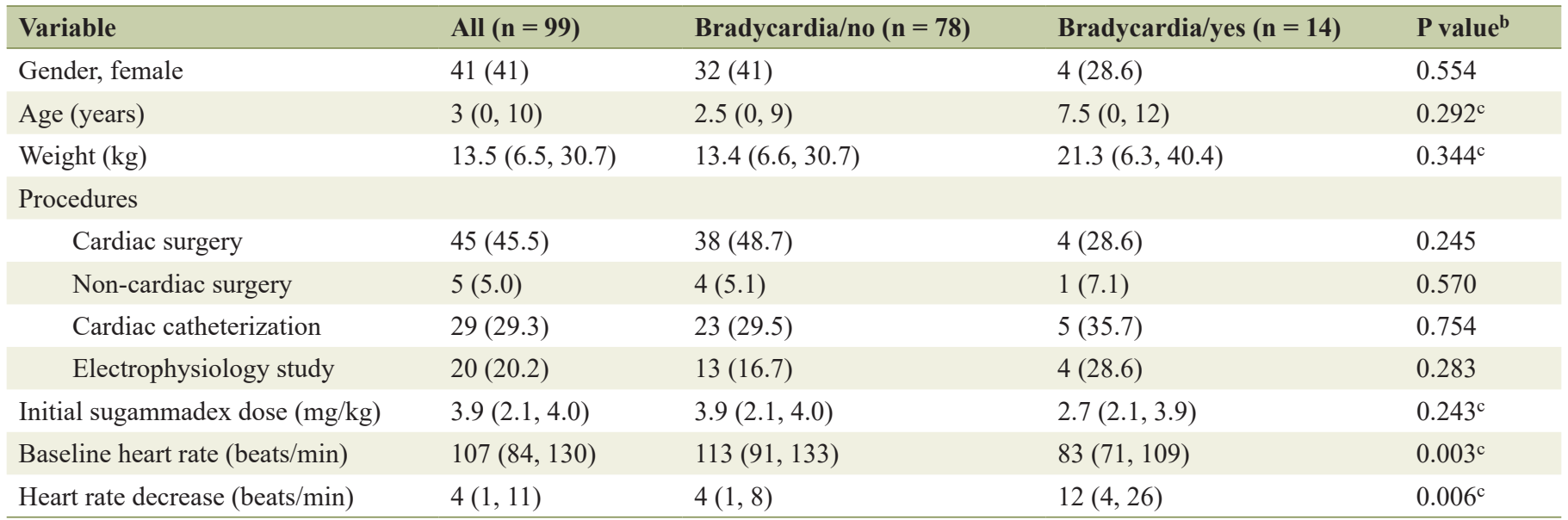

Date presented as $\mathrm{n}(\%)$ or median (IQR). aSeven patients with bradycardia at baseline were eliminated from the bradycardia/yes versus bradycardia/ no comparison. bifference between groups tested using Fisher's exact test. 'Difference between groups tested using Mann-Whitney U test. IQR: interquartile range; $\mathrm{n}$ : number.

The clinically significant adverse effects reported during pre-clinical trials included bradycardia and anaphylaxis. As noted in the FDA-approved package insert, marked bradycardia with the occasional progression to cardiac arrest has been observed within minutes after administration. Despite this significant adverse effect, no mechanism has been proposed and no risk factors identified for this response. Despite its relative safety, anecdotal reports have supported the temporal association of bradycardia or cardiac conduction disturbances following sugammadex administration [7-9, 18]. However, the incidence of bradycardia is lower with sugammadex than with neostigmine with adult studies reporting an incidence of $2 \%$ with limited data regarding the hemodynamic impact of HR changes or the need to treat bradycardia due to clinical compromise $[13,15]$.

In our previous study, we noticed a greater incidence of bradycardia in patients with comorbid cardiac, cardiovascular, and congenital heart diseases. The current study expands on this finding, focusing on the subset of patients with cardiac diseases. Although the incidence of bradycardia was higher than that previously reported in adults or children, none of episodes of bradycardia resulted in clinically significant hemodynamic effects requiring clinical intervention or treatment. The higher incidence noted in this study and our previous study may be

Table 3. Multivariable Regression of Characteristics Associated With Bradycardia $(\mathrm{N}=92)^{\mathrm{a}}$

\begin{tabular}{llll}
\hline Characteristics & Odds ratio & $\mathbf{9 5 \%}$ confidence interval & P value \\
\hline Female & 0.58 & $0.17,1.99$ & 0.383 \\
Age (years) & 1.06 & $0.96,1.17$ & 0.247 \\
Weight $(\mathrm{kg})$ & 1.01 & $0.99,1.04$ & 0.413 \\
Initial sugammadex dose $(\mathrm{mg} / \mathrm{kg})$ & 0.67 & $0.40,1.13$ & 0.132 \\
\hline
\end{tabular}

aSeven patients with bradycardia at baseline were eliminated from multivariable regression analysis.

Table 4. Cardiac Comorbid Conditions in the Study Cohort $(N=99)$

\begin{tabular}{llll}
\hline Cardiac comorbid condition $^{\mathrm{a}}$ & All $(\mathbf{n}=\mathbf{9 9})$ & Bradycardia/no $(\mathbf{n}=\mathbf{7 8})$ & Bradycardia/yes $(\mathbf{n}=\mathbf{1 4})$ \\
\hline Congenital heart disease $^{\mathrm{b}}$ & 72 & 59 & 8 \\
Valvular heart disease (pulmonary or aortic stenosis) & 4 & 3 & 1 \\
Cardiomyopathy & 3 & 3 & 0 \\
Status post heart transplant & 3 & 2 & 1 \\
Arrhythmias & 15 & 9 & 4 \\
No other cardiac comorbidity & 2 & 2 & 0 \\
\hline
\end{tabular}

aSeven patients with bradycardia at baseline were eliminated from the bradycardia/yes versus bradycardia/no comparison. ${ }^{b}$ Atrial septal defect, ventricular septal defect, patent ductus arteriosus, atrioventricular canal, tetralogy of Fallot, hypoplastic left heart syndrome, pulmonary atresia, coarctation of the aorta and truncus arteriosus. 


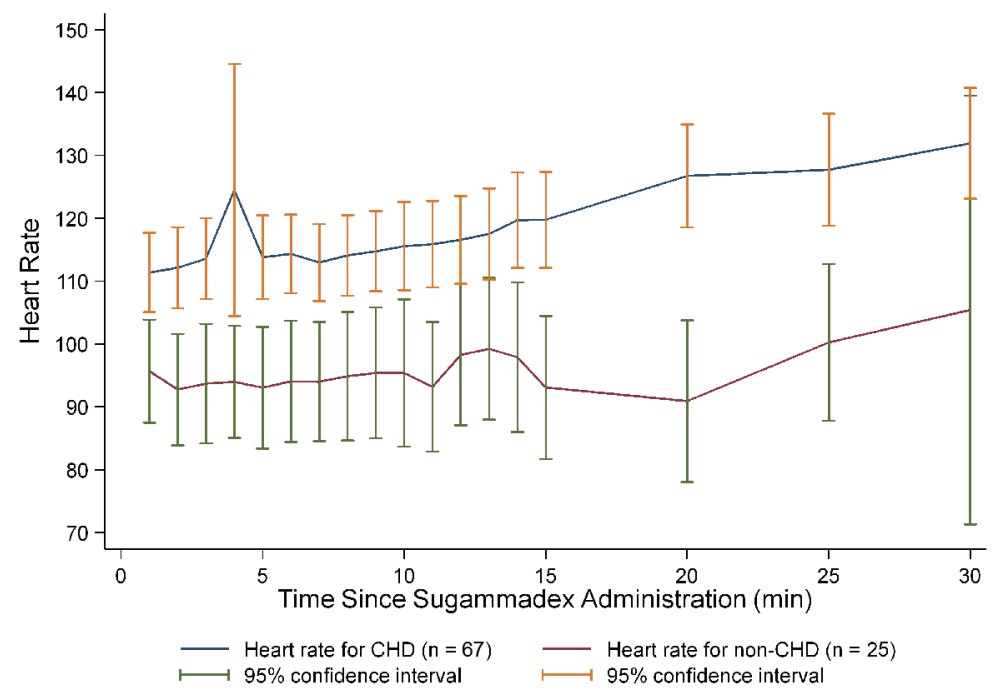

Figure 2. Average heart rate for the first 30 min following sugammadex in patients with comorbid cardiac, cardiovascular, and congenital heart diseases $(n=92)$. Seven patients with bradycardia at baseline were eliminated. CHD: congenital heart disease.

partially explained by our definition of bradycardia as HR less than the fifth percentile for age. Additionally, several patients had bradycardia prior to the administration of sugammadex which may have artificially elevated the incidence.

Patients with comorbid cardiac diseases may be prone to bradycardia related to residual conduction effects of cardiac surgery or cardiopulmonary bypass, among other reasons unrelated to sugammadex administration. However, we noted no association between the specific type of comorbid cardiac disease or the type or the procedure and the risk of bradycardia. As reported in our previous study, there was a trend toward a higher incidence in older patients, those who weighed more and male gender, though this was not statistically significant.

Limitations of the current study include absence of a comparative group of patients receiving neostigmine, to allow a direct comparison of the incidence of bradycardia between the two groups. We did not rigorously control the dosing of sugammadex or the anesthetic technique, including use of other medications which may have impacted HR. Rather, we left the anesthetic technique and the dosing of sugammadex to the discretion of the anesthesia team, which we believe is more applicable to true clinical practice. We did not find an association between the dose of sugammadex and the incidence of bradycardia. As the anesthetic technique was not controlled, we cannot comment on the association or additive effect of sugammadex with other medications known to have a negative chronotropic effect such as propofol or dexmedetomidine.

With these caveats in mind, the current study cohort provides more information regarding bradycardia following the administration of sugammadex. Using a HR less than the fifth percentile for age as the definition of bradycardia, we noted an incidence of bradycardia of $20 \%$ (14\% if patients with baseline bradycardia are excluded) in patients with comorbid cardiac, cardiovascular or congenital heart disease who received sugammadex. No association was noted with any specific type of comorbid cardiac, cardiovascular or congenital heart disease. Furthermore, no treatment was required and no adverse hemo- dynamic effects were noted. In general, treatment would be indicated for clinically significant hypotension, clinical evidence of decreased cardiac output, or severe bradycardia (HR less than 40 beats/min). Future studies are needed to define the effects of other commonly used anesthetic agents and their potential association with bradycardia following the administration of sugammadex.

\section{Acknowledgments}

None to declare.

\section{Financial Disclosure}

None to declare.

\section{Conflict of Interest}

None to declare.

\section{Informed Consent}

IRB approved waiver of consent as noted in methods section.

\section{Author Contributions}

Jordan Arends: data analysis, preparation of first and subsequent drafts. Richard Hubbard: study design, patient enrollment, manuscript review and preparation. Shabana Z. Shafy: data acquisition and analysis, manuscript review and preparation. Mohammed Hakim: data acquisition and analysis, manu- 
script review and preparation. Stephani S. Kim: data analysis, manuscript review and preparation. Dmitry Tumin: data acquisition and analysis, manuscript review and preparation. Joseph D. Tobias: study design, data analysis, manuscript review and preparation.

\section{Data Availability}

Any inquiries regarding supporting data availability of this study should be directed to the corresponding author.

\section{References}

1. Bom A, Hope F, Rutherford S, Thomson K. Preclinical pharmacology of sugammadex. J Crit Care. 2009;24(1):29-35.

2. Hunter JM, Flockton EA. The doughnut and the hole: a new pharmacological concept for anaesthetists. Br J Anaesth. 2006;97(2):123-126.

3. Cammu G, De Kam PJ, Demeyer I, Decoopman M, Peeters PA, Smeets JM, Foubert L. Safety and tolerability of single intravenous doses of sugammadex administered simultaneously with rocuronium or vecuronium in healthy volunteers. Br J Anaesth. 2008;100(3):373-379.

4. Gijsenbergh F, Ramael S, Houwing N, van Iersel T. First human exposure of Org 25969, a novel agent to reverse the action of rocuronium bromide. Anesthesiology. 2005;103(4):695-703.

5. Sorgenfrei IF, Norrild K, Larsen PB, Stensballe J, Ostergaard D, Prins ME, Viby-Mogensen J. Reversal of rocuronium-induced neuromuscular block by the selective relaxant binding agent sugammadex: a dose-finding and safety study. Anesthesiology. 2006;104(4):667-674.

6. Tobias JD. Current evidence for the use of sugammadex in children. Paediatr Anaesth. 2017;27(2):118-125.

7. Bilgi M, Demirhan A, Akkaya A, Tekelioglu UY, Kocoglu H. Sugammadex associated persistent bradycardia. Int J Med Sci Public Health. 2014;3:372-374.

8. King A, Naguib A, Tobias JD. Bradycardia in a Pediat- ric Heart Transplant Recipient: Is It the Sugammadex? J Pediatr Pharmacol Ther. 2017;22(5):378-381.

9. Bhavani SS. Severe bradycardia and asystole after sugammadex. Br J Anaesth. 2018;121(1):95-96.

10. Alsuhebani M, Sims T, Hansen JK, Hakim M, Walia H, Miller R, Tumin D, et al. Heart rate changes following the administration of sugammadex in children: a prospective, observational study. J Anesth. 2020;34(2):238-242.

11. Bonafide CP, Brady PW, Keren R, Conway PH, Marsolo $\mathrm{K}$, Daymont $\mathrm{C}$. Development of heart and respiratory rate percentile curves for hospitalized children. Pediatrics. 2013;131(4):e1150-1157.

12. Welliver M, McDonough J, Kalynych N, Redfern R. Discovery, development, and clinical application of sugammadex sodium, a selective relaxant binding agent. Drug Des Devel Ther. 2009;2:49-59.

13. Carron M, Zarantonello F, Tellaroli P, Ori C. Efficacy and safety of sugammadex compared to neostigmine for reversal of neuromuscular blockade: a meta-analysis of randomized controlled trials. J Clin Anesth. 2016;35:1-12.

14. Koyuncu O, Turhanoglu S, Ozbakis Akkurt C, Karcioglu M, Ozkan M, Ozer C, Sessler DI, et al. Comparison of sugammadex and conventional reversal on postoperative nausea and vomiting: a randomized, blinded trial. J Clin Anesth. 2015;27(1):51-56.

15. Hristovska AM, Duch P, Allingstrup M, Afshari A. The comparative efficacy and safety of sugammadex and neostigmine in reversing neuromuscular blockade in adults. A Cochrane systematic review with meta-analysis and trial sequential analysis. Anaesthesia. 2018;73(5):631-641.

16. Brueckmann B, Sasaki N, Grobara P, Li MK, Woo T, de Bie J, Maktabi M, et al. Effects of sugammadex on incidence of postoperative residual neuromuscular blockade: a randomized, controlled study. Br J Anaesth. 2015;115(5):743-751.

17. Caldwell JE. Clinical limitations of acetylcholinesterase antagonists. J Crit Care. 2009;24(1):21-28.

18. Osaka Y, Shimada N, Satou M, Masuda T, Ando T, Kozono Y, Shimada M. A case of atrioventricular block (Wenckebach type) induced by sugammadex. J Anesth. 2012;26(4):627-628. 